\title{
The 'armed concrete' approach: stent-screw-assisted internal fixation (SAIF) reconstructs and internally fixates the most severe osteoporotic vertebral fractures
}

\author{
Daniela Distefano, ${ }^{1}$ Pietro Scarone, ${ }_{1}^{2}$ Maurizio Isalberti, ${ }^{1}$ Luigi La Barbera, ${ }^{3,4,5}$ \\ Tomaso Villa, ${ }^{4}$ Giuseppe Bonaldi, ${ }^{6}$ Joshua A Hirsch (ㄷ , ${ }^{7}$ Alessandro Cianfoni ${ }^{1,8}$
}

For numbered affiliations see end of article.

\section{Correspondence to} Dr Daniela Distefano, Department of Neuroradiology, Neurocenter of Southern Switzerland, Lugano, Switzerland; daniela.distefano@ eoc.ch

Received 6 July 2020 Revised 21 August 2020 Accepted 24 August 2020 Published Online First 16 September 2020

\section{Linked}

- http://dx.doi.org/10.1136/ neurintsurg-2020-016894

Check for updates

(C) Author(s) (or their employer(s)) 2021. No commercial re-use. See rights and permissions. Published by BMJ.

To cite: Distefano $D$ Scarone $\mathrm{P}$, Isalberti $\mathrm{M}$, et al. J Neurolntervent Surg 2021:13:63-68.

\section{ABSTRACT}

Background The treatment of severe osteoporotic vertebral compression fractures (VCFs) with middlecolumn (MC) involvement, high fragmentation, large cleft and/or pedicular fracture is challenging. Minimally invasive 'stent-screw-assisted internal fixation' (SAIF) can reduce the fracture, reconstruct the vertebral body (VB) and fix it to the posterior elements.

Objective To assess feasibility, safety, technical and clinical outcome of the SAIF technique in patients with severe osteoporotic VCFs.

Methods 80 treated vertebrae were analyzed retrospectively. Severe VCFs were characterized by advanced collapse (Genant grade 3), a high degree of osseous fragmentation (McCormack grade 2 and 3 ), burst morphology with MC injury, pediculo-somatic junction fracture, and/or large osteonecrotic cleft. VB reconstruction was evaluated on postprocedure radiographs and $\mathrm{CT}$ scans by two independent raters. Clinical and radiological follow-ups were performed at 1 and 6 months.

Results SAIF was performed at 28 thoracic and 52 lumbar levels in 73 patients. One transient neurological complication occurred. VB reconstruction was satisfactory in $98.8 \%$ of levels (inter-rater reliability $96 \%, \kappa=1$ ). Follow-up at 1 month was available for $78 / 80$ levels and at 6 months or later (range 6-24, mean 7.9 months) for $73 / 80$ levels. Significant improvement in the Visual Analog Scale score was noted at 1 and 6 months after treatment $(p<0.05)$. Patients reported global clinical benefit during follow-up (Patient'sGlobal Impression of Change Scale $5.6 \pm 0.9$ at 1 month and $6.1 \pm 0.9$ at 6 months). Fourteen new painful VCFs occurred at different levels in 11 patients during follow-up, treated with vertebral augmentation or SAIF. Target-level stability was maintained in all cases.

Conclusions SAIF is a minimally invasive, safe, and effective treatment for patients with severe osteoporotic VCFs with MC involvement.

\section{INTRODUCTION}

Vertebral compression fractures (VCFs) are well described clinical manifestations of osteoporosis. ${ }^{1}$ The severity spectrum of VCFs is broad. Many are mild and stable compression fractures affecting the anterior column (AC) with minor wedge or biconcave deformity, usually treated with conservative therapy. Those more severely affected may be treated with traditional augmentation techniques as VCFs are often associated with acute and chronic pain, physical impairment, disability, and decreased quality of life, with an impact on mortality. ${ }^{2}$

At the other end of the spectrum, osteoporotic VCFs can have a wide variety of troubling appearances. They can be unstable fractures with severe collapse and kyphotic deformity, burst morphology with middle column (MC) involvement and posterior wall retropulsion, pediculo-somatic junction fracture, high-degree of osseous fragmentation, advanced loss of integrity and quality of trabecular and cortical bone, and fractures with large osteonecrotic clefts. In this study, these types of fracture are aggregated and referred to as 'severe VCFs ${ }^{13-5}$ (online supplemental figure 1S).

Vertebral augmentation (VA) is widely used to palliate painful VCFs resistant to conservative treatment. ${ }^{6}$ For poor surgical candidates, these techniques are also an option to treat severe VCFs.

Ideally, however, these severe VCFs would benefit from kyphosis reduction, supported with multicompartmental stabilization to restore axial load-bearing capability of the vertebral body (VB) and arrest fracture progression. ${ }^{7-10}$ Put differently, standard VA, performed either with vertebroplasty or balloon kyphoplasty, obtains cement augmentation of the AC, the anterior two-thirds of the $\mathrm{VB}$, while the MC is usually left non-reinforced, both for technical constraints and for safety measures, to avoid risk of cement leak in the central canal. Thus, the MC, after VA, is seen as a non-augmented 'bare area' (figure 1).

This bare area, commonly neglected with traditional percutaneous techniques, represents a weak point in an augmented vertebra, ${ }^{11}$ especially when already injured in these severe VCFs, and may play a key role in the refracture of the treated level. These refractures may feature posterior wall retropulsion, cleavage and splitting between the augmented AC and the bare MC, focal kyphosis and instability, posing a real treatment challenge ${ }^{12}$ (figure 1).

Furthermore, in severely collapsed VBs, with a high degree of fragmentation, pediculo-somatic fractures, and advanced loss of osseous integrity, standard VA might be unable to obtain significant 


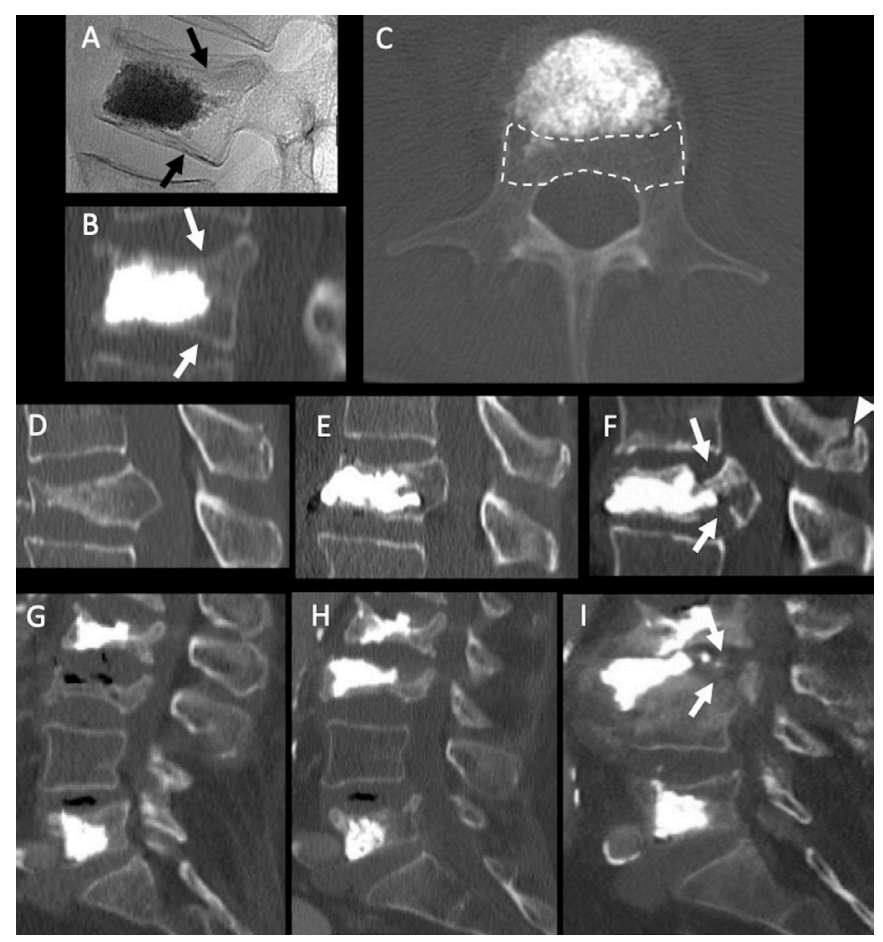

Figure $1(\mathrm{~A}-\mathrm{C})$ The 'bare area' concept: even after technicallysatisfactory vertebral augmentation (VA) the middle column (MC) remains non-augmented, and clearly visible on axial $\mathrm{CT}$, and appears as a non-reinforced bare portion of the vertebral body (area outlined by dashed line on C). The junction between augmented and nonaugmented vertebral body (arrows on $A$ and $B$ ) might represent $a$ weak point, subject to refracture, as in the two clinical examples (D-F and G-I). In both cases, after VA (E and H) of a fracture with MC involvement, refracture occurs at the MC 'bare area' (arrows on F and I), resulting in posterior wall retropulsion, splitting between augmented and non-augmented vertebral body, and focal hyperkyphosis. In (F) the arrowhead points at a spinous process fracture due to kyphotic deformity.

height and kyphosis reduction and cement might disperse in uneven manner, with overall failure to achieve satisfactory stabilization.

These patients are complex and the challenge of treating these severe VCFs is bidirectional. Although VA might represent an undertreatment for severe VCFs, the alternative of open surgical stabilization is invasive and carries risk of failure in patients with poor bone quality from osteoporosis. ${ }^{13}$

Recently, a new minimally invasive augmentation technique, called stent-screw-assisted internal fixation (SAIF) has been proposed for the treatment of severe osteoporotic and neoplastic fractures. ${ }^{14} 15$ This technique includes insertion and balloon expansion of two vertebral body stents (VBSs), followed by placement of percutaneous cannulated and fenestrated pedicular screws in the stents' lumen, and cement augmentation through the screws, representing an 'armed concrete' approach.

The stents obtain and maintain fracture reduction while the pedicle screws anchor the VBS-cement complex to the posterior elements, avoiding its displacement, and act as a bridge across the MC, preserving its integrity from possible collapse and splitting. ${ }^{15}$ Two biomechanical studies provide support for this approach in both neoplastic and osteoporotic models. ${ }^{11} 16$

The purpose of this study was to assess the feasibility, safety, and technical and clinical outcome of VB reconstruction and fixation through the SAIF technique in a cohort of patients with osteoporotic severe VCFs.

\section{MATERIALS AND METHODS Patients}

This is a retrospective analysis of a single-center, prospectively maintained database of a consecutive series of patients with severe thoracolumbar osteoporotic fractures treated with the SAIF technique between August 2015 and October 2018. The VCFs were characterized by one or more of the following morphological features: advanced collapse (Genant grade 3), burst morphology with MC injury, high degree of osseous fragmentation (McCormack comminution grade 2 and 3), ${ }^{4}$ pediculosomatic junction fracture and/or large osteonecrotic cleft. More than one of the above situations could exist in the same patient (online supplemental figure 1S). The study was approved by the local ethics committee. Informed consent was obtained for all procedures. All patients underwent preprocedural spinal CT and/or MRI at the target level, to accurately define the fracture morphology. Decision to treat with SAIF procedure was reached by a multidisciplinary team of clinical specialists involved in the care and treatment of patients with spine problems, including neurosurgeons, neuroradiologists, pain physicians, and physical medicine and rehabilitation physicians.

\section{SAIF procedure}

The SAIF procedural details have been described previously. ${ }^{15}$

All procedures were performed percutaneously, under general anesthesia, with biplanar fluoroscopic guidance (Philips, Allura). Following bilateral trans-pedicular implant of the VBS (DePuySynthes-Johnson\&Johnson) and placement of unilateral or bilateral screws (Injection pin, 2B1 S.R.L., Milan, Italy), cement augmentation was performed through the screw(s) with high-viscosity polymethyl methacrylate (PMMA; Vertaplex HV, Stryker, Kalamazoo, Michigan, USA) under real-time continuous fluoroscopic monitoring.

Adjacent or distant vertebral levels were treated with VA during the same procedure, in cases of multilevel osteoporotic VCFs, or with prophylactic intent, when deemed appropriate by the operator. ${ }^{17}$

Patients were allowed to stand and walk without spinal braces as soon as 3 hours after the procedure, and commonly discharged the same day, in a day-surgery setting.

\section{Assessment of VB reconstruction, complications and follow- up}

VB reconstruction was assessed with postprocedure radiographs and CT scan. CT datasets were reconstructed with a bone algorithm with $3 \mathrm{~mm}$ and $10 \mathrm{~mm}$ thick maximum intensity projection images in the three orthogonal planes, and independently reviewed by a neuroradiologist (AC) and a neurosurgeon (PS). We adopted the same qualitative four-grade scale (poor, fair, good, excellent) previously used in VBS and SAIF studies to assess VB reconstruction, ${ }^{14}{ }^{18}$ based on overall assessment of correct placement and expansion of the implants, cement filling, and VB height restoration.

Poor indicated failure to achieve sufficient augmentation of the AC, whereas excellent indicated appropriate stent expansion, cement filling, and consequent satisfactory height restoration and correct screw(s) positioning. An excellent result would appear as an internal VB prosthesis of the affected VB. Good and excellent ratings were considered satisfactory results. 
Intraprocedural complications, such as potentially significant cement leaks and misplacement of the screws, were recorded.

Patients were followed-up at 1 month and 6 months, with a clinical examination and upright plain radiographs, and then at variable intervals, following clinical practice. For some patients late clinical follow-up was performed over the phone by a physician (DD). When clinically necessary further imaging with CT or MRI was performed during follow-up. The Visual Analog Scale (VAS) pain score (range 0-10) was obtained before the procedure and at 1 month and 6 months after treatment. Patient's Global Impression of Change (PGIC) Scale, featuring a sevenpoint response ((1) extremely worse, (2) much worse, (3) a little worse, (4) no change, (5) a little better, (6) much better, (7) extremely better) ${ }^{19}$ was obtained at 1 and 6 months after treatment.

Imaging follow-up was evaluated to assess refractures, new or worsening spinal deformity of the treated segment, mobilization of the VBS and screw implants, and new vertebral fractures at adjacent levels.

\section{Statistical analyses}

Analyses were conducted using SPSS version 20.0.0 (IBM Corp.). Descriptive statistics for demographic and clinical data were expressed as mean and range or median with IQR. Differences in VAS scores before and after treatment were tested by Wilcoxon test. A p value $<0.05$ was considered statistically significant.

VB reconstruction results were judged according to the following scale: poor, fair, good, or excellent. Excellent and good ratings were considered as satisfactory results. ${ }^{18}$ Cohen's kappa coefficient (к) was used to assess the proportion of agreement of the two independent raters beyond that expected by chance, and the classification by Landis and $\mathrm{Koch}^{20}$ was used to define the agreement level: poor, <0.00; slight, $0.00-0.20$; fair, 0.21-0.40; moderate, $0.41-0.60$; substantial, $0.61-0.80$; or almost perfect, $0.81-1.00$.

\section{RESULTS}

In 73 patients $(21$ men and 52 women; mean age 77.7 years, range 59-98 years), 76 levels with osteoporotic severe VCFs were treated in 73 procedures; then during follow-up, four subsequent severe VCFs at other levels were treated with SAIF, for a total of 80 levels treated with SAIF in 77 procedures. Treated levels were between T3 and L5, 28/80 thoracic (35\%) and 52/80 lumbar (65\%); more specifically 63/80 (79\%) located at the thoracolumbar junction (T10-L2).

A summary of patient's demographic and clinical data and features of VCFs is provided in table 1. Technical results and complications are summarized in online supplemental table $1 \mathrm{~S}$, and follow-up results are summarized in online supplemental table $2 S$.

\section{Technical results}

SAIF procedures were performed as a stand-alone intervention in $78 / 80$ cases, in combination with a percutaneous posterior surgical fixation in one case, and after decompressive laminectomy and posterior surgical fixation in one case presenting with spinal cord compression and new neurological deficit.

VBSs were positioned bilaterally in all cases. Bilateral screws were used in $67 / 80(83.7 \%)$ levels and unilateral screws in $13 / 80$ levels (16.3\%).
Table 1 Characteristics of the study population and features of osteoporotic vertebral compression fractures

\begin{tabular}{|ll|}
\hline Variable & Value \\
\hline Total number. of patients & 73 \\
\hline Sex: M/F & $21 / 52$ \\
\hline Mean age (range) & $77.7(59-98)$ \\
\hline Treated levels (\%) & 80 \\
\hline \multicolumn{1}{|c|}{ Thoracic } & $28 / 80(35 \%)$ \\
\hline Lumbar & $52 / 80(65 \%)$ \\
\hline Thoracolumbar junction (Th10-L2) & $63 / 80(79 \%)$ \\
\hline Fracture morphology, No. of levels (\%) & \\
\hline VB collapse & \\
\hline \multicolumn{1}{|c}{$50 \%$} & $21 / 80(26.3 \%)$ \\
\hline$>50 \%$ & $59 / 80(73.7 \%)$ \\
\hline Genant grade 3 & $51 / 80(63.8 \%)$ \\
\hline Burst & $56 / 80(70 \%)$ \\
\hline McCormack (grade 2 and 3) & $80 / 80(100 \%)$ \\
\hline Pediculo-somatic fractures & $31 / 80(38.8 \%)$ \\
\hline Osteonecrotic cleft & $56 / 80(70 \%)$ \\
\hline VB, vertebral body. & \\
\hline
\end{tabular}

\section{Procedural and periprocedural safety}

Cement leakage was detected at $8 / 80$ levels $(10 \%)$ on postprocedure CT, with an epidural or foraminal location in $3 / 80$ levels $(3.8 \%)$. These patients remained asymptomatic. One patient experienced hypoesthesia and mild motor deficits in the lower limbs 2 days after the procedure. On CT examination there was no evidence of PMMA leaks. Postprocedure MRI demonstrated an intradural T2-hypointense tubular structure. This was hypothesized to represent a venous thrombosis. The symptoms resolved over the next few weeks and MRI performed 3 months postprocedure was normal. No other neurologic periprocedural clinical complications occurred.

\section{VB reconstruction}

VB reconstruction scores assigned by the two readers were respectively excellent at $73 / 80(91.3 \%)$ and $74 / 80(92.5 \%)$ levels, good at $6 / 80(7.5 \%)$ and $5 / 80(6.3 \%)$, fair at 1/80 (1.3\%) for both readers, and poor at none of the treated levels, leading to satisfactory results (excellent or good rating) in 79/80 (98.8\%) cases for both readers. The inter-rater reliability was $96 \%$ with a Cohen's kappa of 1 , indicating perfect agreement among raters.

\section{Follow-up results}

Postprocedure clinical and radiological follow-up was available at 1 month for 78/80 treated levels (72/73 patients) and at 6 months or later (range 6-24 months, mean 7.9 months) for $73 / 80$ levels (68/73 patients).

There was a statistically significant difference in VAS scores before the procedure (median 8, IQR 8-9) versus 1 month (median 3, IQR 1.7-5) and versus 6 months (median 2, IQR $0-3$; Wilcoxon test, all $\mathrm{p}<0.00001)$.

The PGIC Scale was $5.6 \pm 0.9$ at 1 month and $6.1 \pm 0.9$ at 6 months, indicating a very positive patient's subjective global clinical impact.

No cases of stent or screw dislocation were seen until the last available follow-up.

Osseous subsidence of the treated VB around the VBS-cement complex was observed during follow-up in 16/80 levels (20\%), 


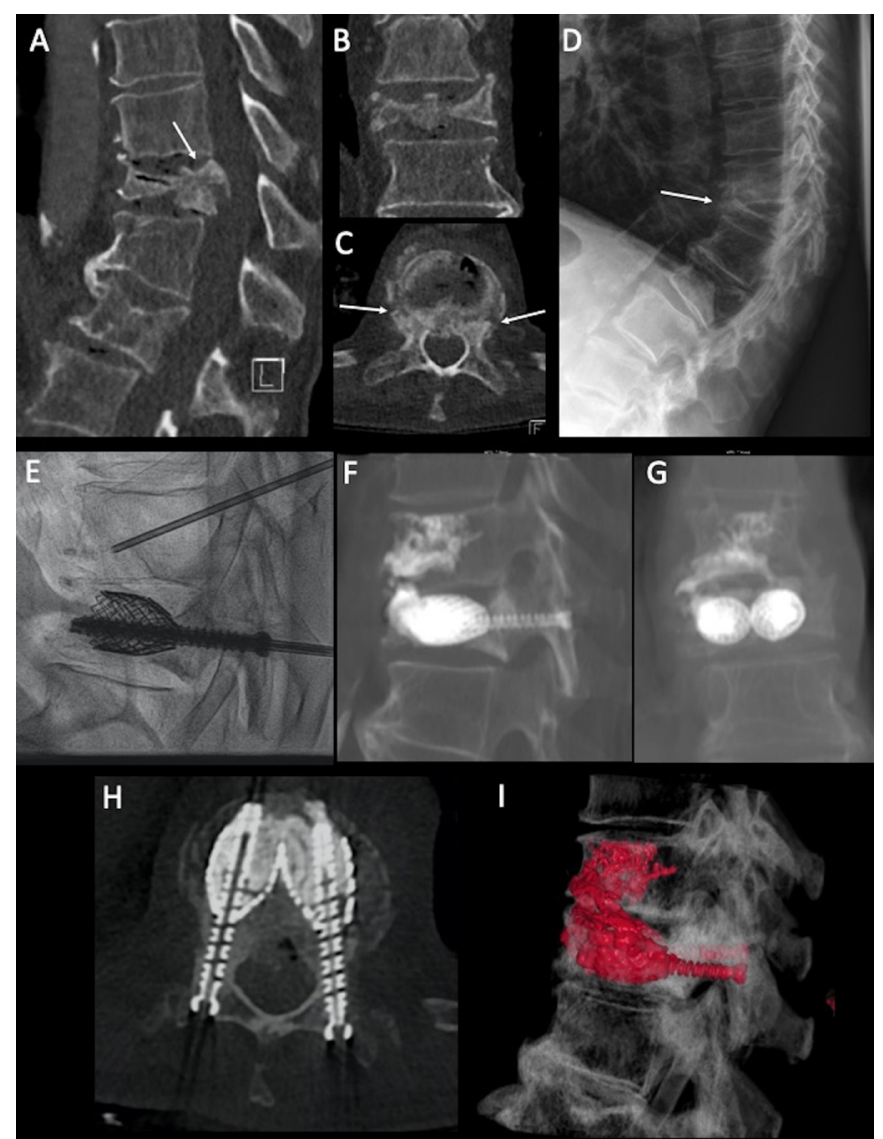

Figure 2 (A-D) Osteoporotic severe fracture at a junctional level (T10), with burst morphology, high degree of fragmentation, middle column involvement (arrow on A), bilateral fracture at the pediculosomatic junction (arrows on C), and severe collapse deformity with hyperkyphosis (arrow on D). (E) A fluoroscopic image after vertebral body stent expansion and fracture reduction, and pedicular screw insertion. (F-I) The reformatted CT scan after stent-screw-assisted internal fixation, with cement filling of the stents, and vertebroplasty at the adjacent cranial level, which was also fractured, with a cleft along the inferior disc endplate.

with mild to moderate secondary VB height loss, without onset of new symptoms, and no re-treatment or surgical intervention was necessary at the target level.

Eleven patients during follow-up required a new procedure for a total of 14 new painful VCFs at adjacent or distant levels. Ten levels were treated with vertebroplasty, whereas four, with a new severe VCF, were treated with SAIF.

\section{DISCUSSION}

In this series of patients with osteoporotic severe VCF, characterized by a high degree of collapse, osseous fragmentation, burst morphology with MC involvement, pedicular fracture, and/or large osteonecrotic cleft, SAIF proved to be a feasible and safe minimally invasive procedure for $\mathrm{VB}$ reconstruction and stabilization (figures 2 and 3), with good clinical outcome, and durable results at follow-up.

Most osteoporotic VCFs are stable lesions, with AC injury, and augmentation can reinforce the $\mathrm{VB}$ and prevent further collapse. Severe VCFs, however, almost invariably feature MC involvement. In these situations, $\mathrm{VA}$, leaving the $\mathrm{MC}$ as a nonaugmented bare area (figure 1), might represent undertreatment, while surgical fixation is invasive, carries high rate of fixation
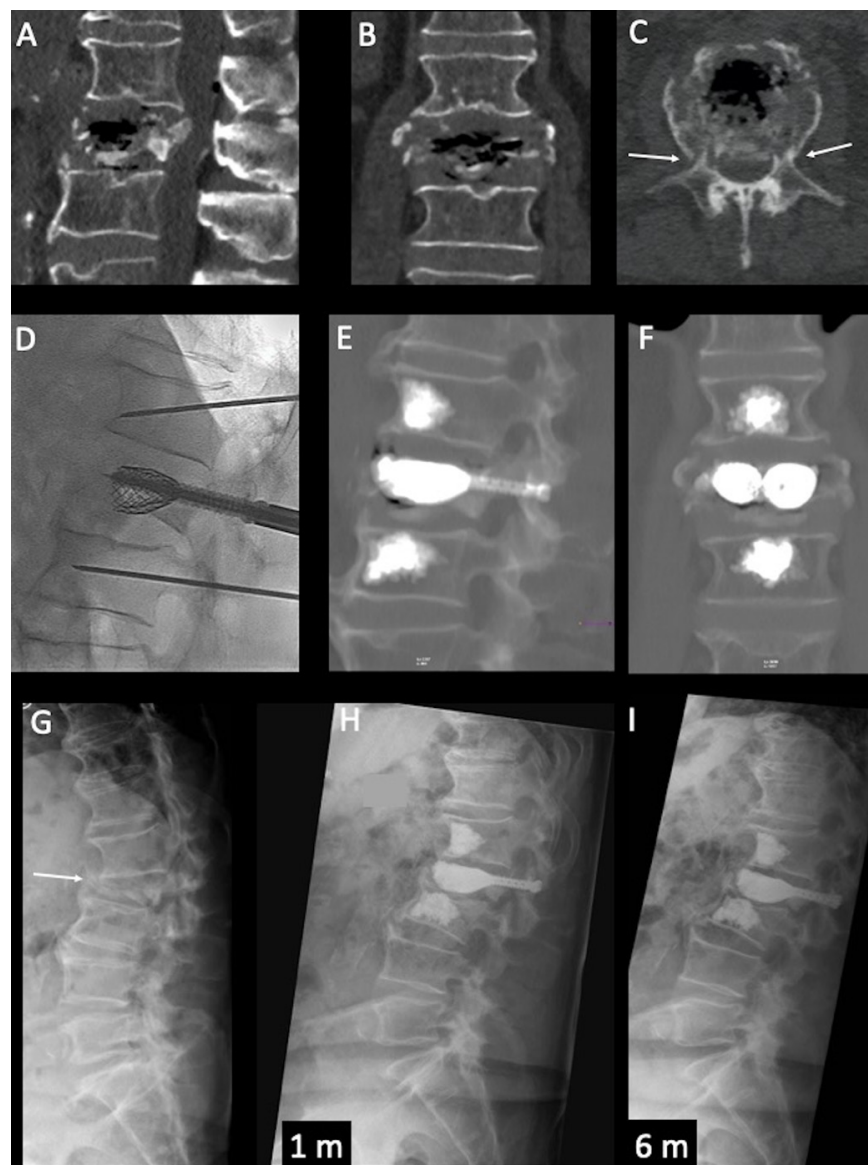

Figure 3 (A-C) An extremely severe fragmentation of an L2 vertebral fracture, with middle column involvement, large air-filled cleft, and bilateral pedicular fracture (arrows on C). (D) A fluoroscopic image of the stent-screw-assisted internal fixation (SAIF) set-up, with stents expanded, screws inserted, and small-caliber cannulae inserted at adjacent levels for prophylactic augmentation; ( $E$ and F) Post-SAIF CT scan, showing reconstruction of the vertebral body and the screws' internal fixation. (G) Standing plain film, preprocedure, with severe collapse and focal kyphosis (arrow), which appears markedly reduced at 1 -month follow-up $(\mathrm{H})$, with stable results at the 6 -month standing film (I).

failure in osteoporotic patients, and might be contraindicated in fragile and elderly patients. ${ }^{12} 1321$

Severe VCFs pose a treatment challenge not only for pain palliation, but also for stabilization, kyphosis correction, and central canal encroachment.

This is the first reported series of osteoporotic severe VCFs treated with the SAIF technique, with the intent to obtain a $360^{\circ}$ non-fusion internal VB fixation, with an armed concrete approach. VBS is used to restore VB height and obtain kyphosis correction, scaffold the VB, help cement containment, and reinforce the AC. The addition of the screws guarantees the anchoring of the VBS-PMMA implant to the posterior elements, preventing their mobilization, and has the potential to reinforce and bridge the MC and the frequently associated pediculosomatic junction fractures.

The structure offered by the metallic stents and the screws, together with the PMMA cement filling, appears as a VB prosthesis, in these vertebrae with very poor or highly destroyed bone stock (figures 2 and 3 ). 
The procedure was safe in this series, with only one patient experiencing a transient self-resolving neurological complication, the nature of which was not readily relatable to technical aspects of the procedure itself.

We observed a $3.8 \%$ rate of cement leaks in an epidural or foraminal location, but those patients remained asymptomatic. In light of the anatomic complexity of these fractures, this leakage rate seems reasonable in comparison with patients with more typical fractures.

The main purpose of the SAIF procedure is vertebral reconstruction to restore axial load-bearing capabilities of the VB. ${ }^{15}$ Technically, the construct was judged as satisfactory when appropriate placement of the devices, VBS expansion, and cement filling restored VB height and achieved reconstruction of the fragmented $\mathrm{VB}$, appearing as a $360^{\circ}$ non-fusion internal fixation of the affected VB. The reconstruction was judged satisfactory (good or excellent) in $98.8 \%$ of cases by the two raters, with perfect inter-rater agreement.

Follow-up was available for 78/80 levels at 1 month, and for $73 / 80$ levels at 6 months and beyond, up to 24 months. The stability of the construct was maintained in all cases until the last available follow-up without VBS mobilization. Migration of stents in highly osteoporotic vertebral bodies is possible, with potential risk of lumbar plexus or great vessels damage. ${ }^{22}$ In patients with extensive VB fragmentation (figure 3 ), it is certainly conceivable that the stents might mobilize in absence of an intact VB cortical shell, as reported in a previous SAIF series in extreme neoplastic osteolysis. ${ }^{14}$

The screw anchoring may represent a means of avoiding VBS mobilization in conjunction with other technical measures such as a PMMA bridge, cement interdigitation, and optimized implant(s) positioning (figure 2).

In this series screws were positioned bilaterally in the majority of cases (67/80 levels). We recommend bilateral screw fixation to anchor the VBS-cement implant to the posterior elements, thereby reducing the risk of VBS mobilization whenever possible. If this is not possible (ie, pedicle fragmentation or small pedicular diameter) a 'kissing configuration' of the VBSs should be obtained to ensure the creation of a PMMA bridge between the two VBSs.

All screws were correctly positioned within the pedicles and in the VBS under fluoroscopic guidance, with no screw loosening observed at follow-up. The screws implanted in the SAIF technique are not connected to posterior fixation rods; thus, there are no high loadings that could predispose to screw loosening or failure, ${ }^{11} 16$ thus differing from the changes in posterior surgical instrumentation. $^{23}$

Following VA, refracture of the treated VB is a well-known event, with an incidence ranging from $3.2 \%$ to $63 \%$ and a cumulative rate of $10.2 \% .^{24}{ }^{25}$ In cement-only VA, subsidence may determine refractures of the non-augmented $\mathrm{MC}$ at the junction with the augmented $\mathrm{AC},{ }^{12}$ with collapse and retropulsion of the posterior wall, eventually associated with catastrophic splitting and separation between the augmented anterior portion of the $\mathrm{VB}$ and the MC, accompanied by focal kyphotic deformity (figure 1).

Although largely under-reported in the literature, these dramatic events pose a real therapeutic challenge. This complication, as previously stated, could be biomechanically explained by the high strain gradient across the augmented AC and the weaker unprotected MC, leading to local intensification effects ${ }^{11}$; moreover, the higher load transfer to the stiff AC, ${ }^{11}$ reduces the mechanical stimulus on the $\mathrm{MC}$, leading to bone resorption.
At imaging follow-up we observed phenomena of osseous remodeling around the VBS-PMMA cast, with features of mild refracture/subsidence in 16/80 treated levels (20\%), with mild to moderate secondary VB height loss, and in a few cases, documented by CT, even mild increase in posterior wall retropulsion but without splitting or increased kyphosis. One might consider subsidence of the endplates as nearly physiological changes after SAIF - namely, because the surrounding fractured and weakened bone of the VB, on weight-bearing loading, remodels and might undergo resorption phenomena against the new rigid internal scaffold, represented by VBS with PMMA, but usually this does not have clinical significance. Indeed, no patients in this series required re-intervention, or surgical salvage at the target level.

These results seem to confirm recently published biomechanical data on a finite-element analysis of the SAIF technique applied to a lumbar osteoporotic spinal model. ${ }^{11}$ SAIF was significantly more effective than simple VA in reducing the median strain distribution across the MC, especially on the superior endplate and on the posterior wall. ${ }^{11}$

Clinical results revealed a meaningful positive effect on back pain shown by significant reduction in VAS at 1 month after the procedure, which was sustained at the 6-month follow-up. Moreover, the patients judged that the procedure had a very positive impact on their situation, as reflected by high PGIC scores at 1 - and 6-months' follow-up.

During follow-up 11 patients required a new procedure to treat new fractures, at adjacent or distant levels, which were painful or causing local hyperkyphosis. Seven cases were treated with simple vertebroplasty, under local anesthesia, while in four cases, with a severe VCF, a new SAIF procedure was performed.

The causes of postprocedure fractures are debated: the stiffness of the SAIF construct is a possible cause, but it should be considered that all these patients presented with extremely severe VCFs, suggesting advanced osteoporosis, many presented with multilevel VCFs, and 79\% of the target level VCFs were at a thoracolumbar junctional level, characterized by significant focal kyphosis, therefore at particular biomechanical risk. ${ }^{26}$ We strongly recommend an appropriate medical therapy to correct osteoporosis, which represents a major risk factor in the development of new VCFs.

In this series of severe VCF, preoperative pedicular fractures were present in $31 / 80$ levels $(38.8 \%)$. Some authors have reported cement augmentation of the pedicles and pediculosomatic junction, the so-called pediculoplasty, ${ }^{27}$ but it should be considered that main loadings at the level of the pedicles and pediculo-somatic junction are in bending, thus involving local tensile loads on the bone, while PMMA is known to have optimal resistance to compressive rather than to tensile loads. ${ }^{28}$ Pedicular screws offer the advantage of internally scaffolding the pedicles, while undergoing relatively low bending stresses, ${ }^{11}$ even when pedicular bone properties are totally compromised. ${ }^{16}$ To confirm these concepts, pedicular or pediculo-somatic junction fractures have not shown dislocation or pseudoarthrosis in the current study at the latest follow-up.

The presence of an intravertebral cleft is associated with significant VB height reduction and is an important risk factor that might prevent osseous healing and might promote the progression of collapse. ${ }^{29}$ The dynamic instability, with subsequent hypermobility at the fractured level, may lead to gradual retropulsion of bony fragments into the spinal canal, with the risk of possible neurological complications. In our series of severe VCFs, a cleft was present in 56/80 levels (70\%), associated in many cases with extremely poor bone stock remaining in the VB. In these cases the VBS recreates the internal structure of the 
$\mathrm{VB}$, and favors a predictable and uniform cement distribution within the stents. ${ }^{15}$ Furthermore, as demonstrated by Venier $e t$ al, 'armed kyphoplasty' with rigid VB distraction devices, such as VBSs, deal safely with the posterior wall retropulsion, when present, by exploiting a ligamentotaxis effect to achieve indirect central canal decompression. ${ }^{30}$

Finally, in cases with signs of gross instability, or when a decompressive laminectomy is necessary, SAIF can be combined with a posterior surgical approach, as occurred in $2 / 80$ cases in this series.

\section{Study limitations}

The study is retrospective but based on a prospectively maintained database. The patients included in this study were evaluated by a multidisciplinary team and considered poor candidates both for VA, because of the severity of their VCF, and for surgical stabilization, either due to poor bone quality or their clinical conditions. In these cases, SAIF was a unique solution to a realworld treatment challenge, which mitigates the limit of a lack of a control group. Of note, the specific vertebral body stents and percutaneous fenestrated screws lack US Food and Drug Administration approval and these procedures have thus far all been performed in Europe.

\section{CONCLUSION}

Our results support the SAIF technique as a minimally invasive procedure of internal stabilization to treat patients with severe osteoporotic VCFs with MC involvement. In our study SAIF proved to be a feasible, safe, and effective treatment to stabilize the VB and to palliate pain, with durable results at follow-up. It might therefore be considered as a valuable option to a more invasive corpectomy, as a stand-alone intervention or in combination with a posterior surgical approach of stabilization. The use of this procedure in clinical practice is supported by a strong biomechanical rationale. Further multicenter prospective data are necessary to confirm our results.

\section{Author affiliations}

${ }^{1}$ Department of Neuroradiology, Neurocenter of Southern Switzerland, Lugano, Switzerland

${ }^{2}$ Department of Neurosurgery, Neurocenter of Southern Switzerland, Lugano, Switzerland

${ }^{3}$ Department of Mechanical Engineering, Polytechnique Montréal, Montreal, Québec, Canada

${ }^{4}$ Laboratory of Biological Structure Mechanics, Department of Chemistry, Materials and Chemical Engineering "Giulio Natta", Politecnico di Milano, Milan, Italy

${ }^{5}$ Sainte-Justine Clinical Hospital Center, Montréal, Quebec, Canada

${ }^{6}$ Department of Neurosurgery, Casa di Cura Igea, Milano, Lombardia, Italy

${ }^{7}$ Department of Radiology, Massachusetts General Hospital, Harvard Medical School, Boston, Massachusetts, USA

${ }^{8}$ Department of Interventional and Diagnostic Neuroradiology, Inselspital University Hospital Bern, Bern, Switzerland

\section{Twitter Joshua A Hirsch @JoshuaAHirsch}

Contributors All authors contributed substantially to the conception or design of the presented work. Acquisition of data: DD, AC, PS, MI. Analysis and interpretation of data: all authors. Drafting the article: all authors. Critically revising the article: JAH, AC. Statistical analysis: DD. Study supervision: AC, TV, GB, JAH.

Funding The authors have not declared a specific grant for this research from any funding agency in the public, commercial or not-for-profit sectors.

Competing interests None declared.

Patient consent for publication Not required.

Ethics approval Ethics committee of Canton Ticino (ID 14-136).
Provenance and peer review Not commissioned; externally peer reviewed.

Data availability statement All data relevant to the study are included in the article or uploaded as supplementary information.

\section{ORCID iD}

Joshua A Hirsch http://orcid.org/0000-0002-9594-8798

\section{REFERENCES}

1 Ensrud KE, Schousboe JT. Vertebral fractures. N Engl J Med Overseas Ed 2011;364:1634-42.

2 Bliuc D, Nguyen ND, Milch VE, et al. Mortality risk associated with low-trauma osteoporotic fracture and subsequent fracture in men and women. JAMA 2009;301:513.

3 Denis F. The three column spine and its significance in the classification of acute thoracolumbar spinal injuries. Spine 1983:8:817-31.

4 McCormack T, Karaikovic E, Gaines RW. The load sharing classification of spine fractures. Spine 1994;19:1741-4.

5 Genant HK, Wu CY, van Kuijk C, et al. Vertebral fracture assessment using a semiquantitative technique. J Bone Miner Res 1993;8:1137-48.

6 Evans AJ, Kip KE, Brinjikji W, et al. Randomized controlled trial of vertebroplasty versus kyphoplasty in the treatment of vertebral compression fractures. J Neurointerv Surg 2016:8:756-63.

7 Firanescu C, Lohle PN, de Vries J, et al. A randomised sham controlled trial of vertebroplasty for painful acute osteoporotic vertebral fractures (VERTOS IV). Trials 2011:12:93.

8 Wardlaw D, Cummings SR, Van Meirhaeghe J, et al. Efficacy and safety of balloon kyphoplasty compared with non-surgical care for vertebral compression fracture (free): a randomised controlled trial. Lancet 2009;373:1016-24.

9 Klazen CAH, Lohle PNM, de Vries J, et al. Vertebroplasty versus conservative treatment in acute osteoporotic vertebral compression fractures (Vertos II): an open-label randomised trial. Lancet 2010:376:1085-92.

10 Clark W, Bird P, Gonski P, et al. Safety and efficacy of vertebroplasty for acute painful osteoporotic fractures (vapour): a multicentre, randomised, double-blind, placebocontrolled trial. Lancet 2016;388:1408-16.

11 La Barbera L, Cianfoni A, Ferrari A, et al. Stent-screw assisted internal fixation of osteoporotic vertebrae: a comparative finite element analysis on SAIF technique. Front Bioeng Biotechnol 2019;7:291

12 Gan M, Zou J, Zhu X, et al. Balloon kyphoplasty for osteoporotic spinal fractures with middle column compromise. Injury 2014;45:1539-44.

13 Abudou M, Chen X, Kong X, et al. Surgical versus non-surgical treatment for thoracolumbar burst fractures without neurological deficit. Cochrane Database Syst Rev 2013:CD005079.

14 Cianfoni A, Distefano D, Scarone P, et al. Stent screw-assisted internal fixation (SAIF): clinical report of a novel approach to stabilizing and internally fixating vertebrae destroyed by malignancy. J Neurosurg Spine 2019:507-18.

15 Cianfoni A, Distefano D, Isalberti M, et al. Stent-screw-assisted internal fixation: the SAIF technique to augment severe osteoporotic and neoplastic vertebral body fractures. J Neurointerv Surg 2019;11:603-9.

16 La Barbera L, Cianfoni A, Ferrari A, et al. Stent screw-assisted internal fixation (SAIF) of severe lytic spinal metastases: a comparative finite element analysis of the SAIF technique. World Neurosurg 2019;128:e370-7.

17 Kobayashi N, Numaguchi Y, Fuwa S, et al. Prophylactic vertebroplasty: cement injection into non-fractured vertebral bodies during percutaneous vertebroplasty. Acad Radiol 2009;16:136-43

18 Cianfoni A, Distefano D, Pravatà E, et al. Vertebral body stent augmentation to reconstruct the anterior column in neoplastic extreme osteolysis. J Neurointerv Surg 2019;11:313-8.

19 Hurst $\mathrm{H}$, Bolton J. Assessing the clinical significance of change scores recorded on subjective outcome measures. J Manipulative Physiol Ther 2004;27:26-35.

20 Landis JR, Koch GG. The measurement of observer agreement for categorical data. Biometrics 1977;33:159

21 Cianfoni A, Giamundo M, Pileggi $M$, et al. Spinal instrumentation rescue with cement augmentation. AJNR Am J Neuroradiol 2018;39:1957-62.

22 Klezl Z, Majeed H, Bommireddy R, et al. Early results after vertebral body stenting for fractures of the anterior column of the thoracolumbar spine. Injury 2011;42:1038-42.

23 La Barbera L, Galbusera F, Wilke H-J, et al. Preclinical evaluation of posterior spine stabilization devices: can we compare in vitro and in vivo loads on the instrumentation? Eur Spine J 2017;26:200-9.

24 Fribourg D, Tang C, Sra P, et al. Incidence of subsequent vertebral fracture after kyphoplasty. Spine 2004;29:2270-6.

25 Uppin AA, Hirsch JA, Centenera LV, et al. Occurrence of new vertebral body fracture after percutaneous vertebroplasty in patients with osteoporosis. Radiology 2003:226:119-24.

26 Ananthakrishnan D, Berven S, Deviren V, et al. The effect on anterior column loading due to different vertebral augmentation techniques. Clin Biomech 2005;20:25-31.

27 Eyheremendy EP, De Luca SE, Sanabria E. Percutaneous pediculoplasty in osteoporotic compression fractures. J Vasc Interv Radiol 2004;15:869-74. 
28 Provenzano MJ, Murphy KPJ, Riley LH. Bone cements: review of their physiochemical and biochemical properties in percutaneous vertebroplasty. AJNR Am J Neuroradiol 2004;25:1286-90.

29 Muratore M, Ferrera A, Masse A, et al. Osteoporotic vertebral fractures: predictive factors for conservative treatment failure. A systematic review. Eur Spine J 2018:27:2565-76.
30 Venier A, Roccatagliata L, Isalberti M, et al. Armed kyphoplasty: an indirect central canal decompression technique in burst fractures. AJNR Am J Neuroradiol 2019;40:1965-72 